\title{
Aerodynamic analysis and control mechanism design of cycloidal wind turbine adopting active control of blade motion
}

\author{
In Seong Hwang*, Yun Han Lee* and Seung Jo Kim** \\ School of Mechanical and Aerospace Engineering, \\ Seoul National University, Korea 151-742
}

\begin{abstract}
This paper describes the cycloidal wind turbine, which is a straight blade vertical axis wind turbine using the cycloidal blade system. Cycloidal blade system consists of several blades rotating about an axis in parallel direction. Each blade changes its pitch angle periodically. Cycloidal wind turbine is different from the previous turbines. The wind turbine operates with optimum rotating forces through active control of the blade to change pitch angle and phase angle according to the changes of wind direction and wind speed. Various numerical experiments were conducted to develop a small vertical axis wind turbine of $1 \mathrm{~kW}$ class. For this numerical analysis, the rotor system equips four blades consisting of a symmetric airfoil NACA0018 of $1.0 \mathrm{~m}$ in span, $0.22 \mathrm{~m}$ in chord and $1.0 \mathrm{~m}$ in radius. A general purpose commercial CFD program, STAR-CD, was used for numerical analysis. PCL of MSC/PATRAN was used for efficient parametric auto mesh generation. Variables of wind speed, pitch angle, phase angle and rotating speed were set in the numerical experiments. The generated power was obtained according to the various combinations of these variables. Optimal pitch angle and phase angle of cycloidal blade system were obtained according to the change of the wind direction and the wind speed. Based on data obtained from the above analysis, control device was designed. The wind direction and the wind speed were sensed by a wind indicator and an anemometer. Each blades were actuated to optimal performance values by servo motors.
\end{abstract}

Key Word : Vertical axis wind turbine, cycloidal wind turbine, cycloidal blade system, straight blade, active control

\section{Introduction}

Recently, the need for development of renewable energy such as wind power has been increasing because of the exhaustion of the earth's natural resources including oil and the effectuation of the Kyoto Protocol of UNFCCC (United Nations Framework Convention on Climate Change). The survey on demand in USA and Europe showed that approximately $12 \%$ of the global power generation will be substituted with wind power by 2020. In Denmark, wind power covers nearly $20 \%$ of their power consumption. In China, wind energy occupies only $0.1 \%$ now; however, they are developing commercial wind power system by cooperation with advanced countries such as Spain. Small scale home use wind power systems are also developing for the people in the western regions which are insufficient in electrical energy.

* Research assistant

** Professor, Director of Flight Vehicle Research Center, corresponding author
E-mail : sjkim@snu.ac.kr
Tel : 02-880-7388
Fax : 02-880-1918 

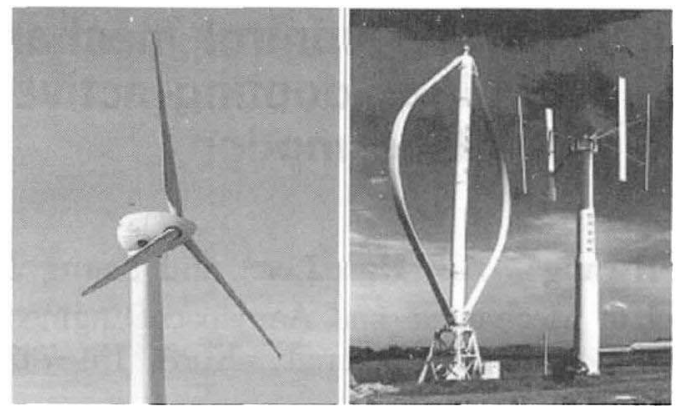

Fig. 1. Classification of wind turbine according to rotating axis type

Figure 1 shows two kinds of wind turbine according to the type of rotating axis: Horizontal Axis Wind Turbine (HAWT) and Vertical Axis Wind Turbine (VAWT). HAWT has a rotating axis parallel to the wind direction, on the other hand, VAWT's rotating axis is vertical to the wind direction. Moreover, two types of wind turbine are exists in VAWT: Savonius type using drag force and Darrieus type using lift force.

Comparing with HAWT, VAWT has remarkable advantages [1]. VAWT is less sensitive to turbulence or change of wind, maintenance and access are easier because generator can be located at the bottom of the rotating axis and the low noise level is low. Relating with these characteristics, VAWT has good potential in the future market; in particular, small wind turbines in urban environment of office buildings, public institutions, large housing complex and private houses.

In this paper, aerodynamic performance was analyzed and blade control mechanism was designed for development of VAWT using the cycloidal blade system. The developing VAWT consists of straight blades and control mechanism, responding actively to the change of wind direction and wind speed.

\section{Cycloidal Wind Turbine}

Cycloidal Wind Turbine (CWT) adopts the cycloidal blade system to the classical VAWT [2, 3]. Figure 2 shows the cycloidal blade system that has been studied at the University of Washington, NACA, etc. since the early 1900s [4-6]. This system produces thrust by varying the cyclic pitch angle of several blades rotating in the direction parallel to the rotating axis [7]. CWT using the cycloidal blade system differs from the classical Darrieus rotor employing blades of fixed pitch angle. CWT maintains efficient power generation by active blade control according to the change of wind direction and wind speed; in particular, pitch angle and phase angle can be controlled during rotor rotation. Figure 3 shows a whole picture of the developing CWT, and Table 1 presents geometric specifications of $1 \mathrm{~kW}$ class wind turbine.

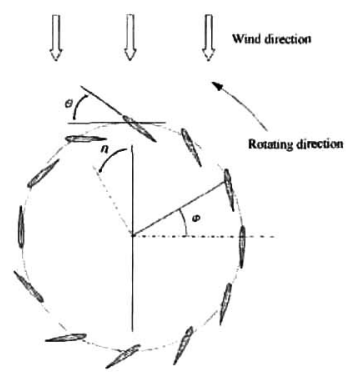

Fig. 2. Cycloidal blade system

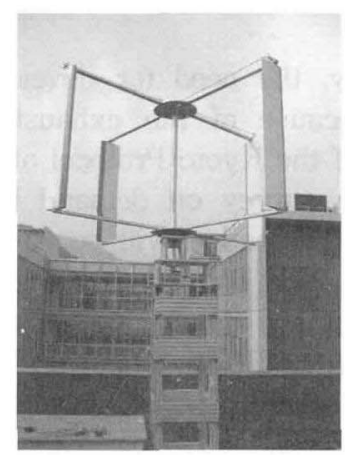

Fig. 3. Cycloidal wind turbine 
Table 1. Geometric characteristics of cycloidal wind turbine

\begin{tabular}{|c|c|}
\hline Parameter & Value \\
\hline \hline Number of blades & 4 \\
\hline Airfoil & NACA0018 \\
\hline Radius of rotor & $1.0 \mathrm{~m}$ \\
\hline Span length of blade & $1.0 \mathrm{~m}$ \\
\hline Chord length of blade & $0.22 \mathrm{~m}$ \\
\hline
\end{tabular}

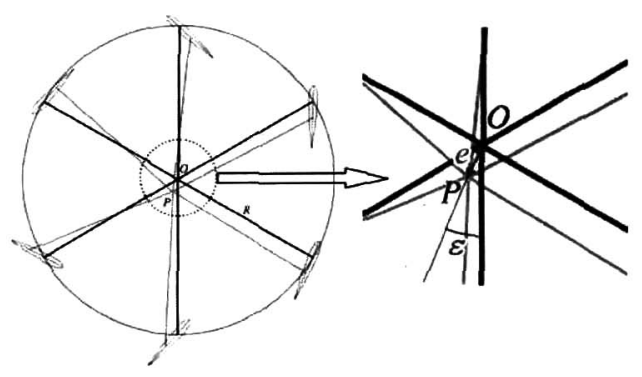

Fig. 4. Basic concept of CWT control mechanism

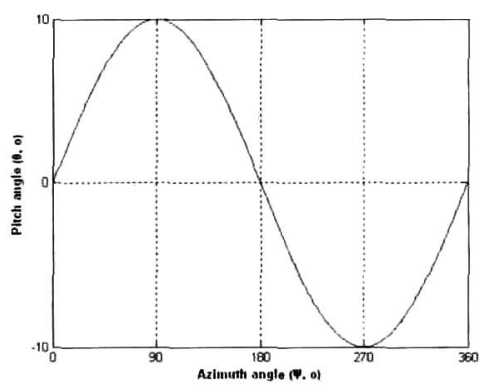

Fig. 5. Pitch angle variation of CWT

In Figure 2, when the wind blows from the upper part to the lower part, the rotor rotates in the counter clockwise direction. The pitch angle, $\theta$ is the angle between the tangent line and the camber line of blade. This angle is varied periodically and maximized when the azimuth angle, $\phi$ is $90^{\circ}$ and $270^{\circ}$ at the setting in Fig. 2. Maximum pitch angle is defined at this azimuth angle position. The phase angle, $n$ is defined as the angle between the wind blowing direction and the line connecting the two positions of the maximum pitch angle. The phase angle is increased in the counter clockwise direction. Figure 4 shows the basic concept of the control mechanism. The magnitude of eccentricity, $e$ is defined as the distance from the center of rotation $O$ to the eccentricity point $P$, as shown in Fig. 4. The phase angle of eccentricity, $\mathcal{E}$ is defined as the angle between the line $O P$ and the vertical line. The magnitude and the phase angle of the eccentricity are used to adjust the pitch angle and the phase angle of the rotor. Figure 5 illustrates pitch angle variation according to the azimuth angle when the maximum pitch angle is $10^{\circ}$ and the phase angle is $0^{\circ}$.

\section{Aerodynamic Performance Analysis of CWT}

To calculate the power generation of CWT according to the change of wind direction and wind speed a commercial CFD program, STAR-CD is used. Moving mesh method is applied for the simulation of rotor rotation and periodic blade pitch variation. Figure 6 shows the CFD mesh in the rotor domain, and Table 2 provides some parameters for this CFD analysis model.

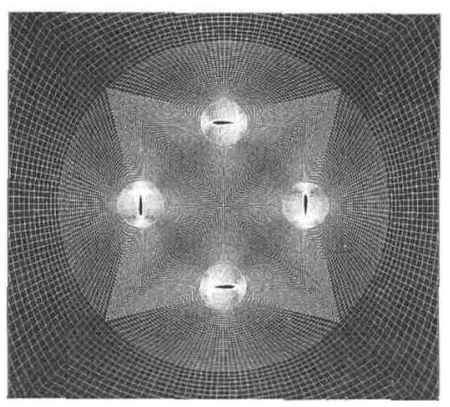

Table 2. CFD analysis model

\begin{tabular}{|c|c|}
\hline Parameter & Value \\
\hline \hline Analysis type & $2-\mathrm{D}$ transient \\
\hline Moving mesh type & ASI \\
\hline Mesh type & Structured (8 node hexahedral) \\
\hline Turbulence model & $k-\mathcal{E}$ hiah Revnolds \\
\hline Total number of cells & 41.472 \\
\hline Rotating anqle per time step & $1.8^{\circ}$ \\
\hline Number of time steps & 1000 \\
\hline
\end{tabular}

Fig. 6. CFD mesh in rotor domain 


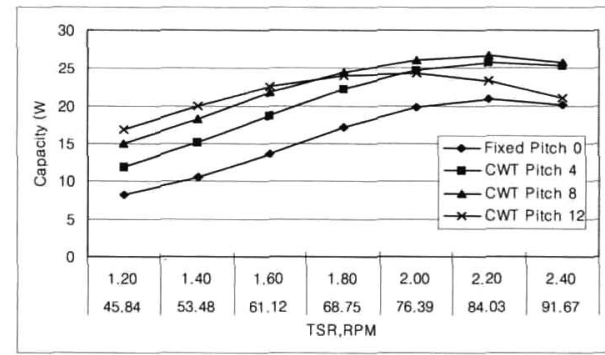

Fig. 7. Power generation according to rotating speed (Wind speed $4 \mathrm{~m} / \mathrm{s}$ )

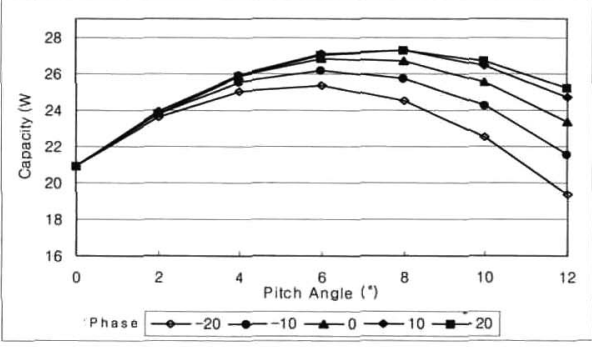

Fig. 9. Power generation according to pitch angle and phase angle (Wind speed $4 \mathrm{~m} / \mathrm{s}$ )

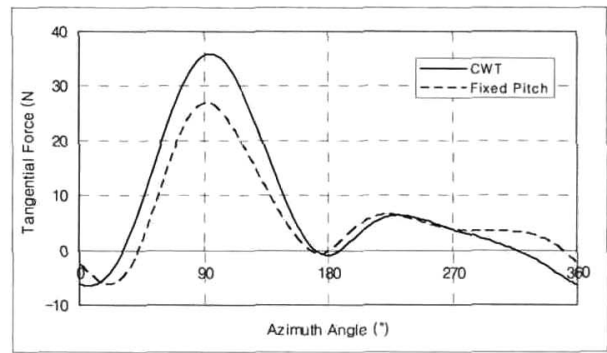

Fig. 11. Tangential force of each blade

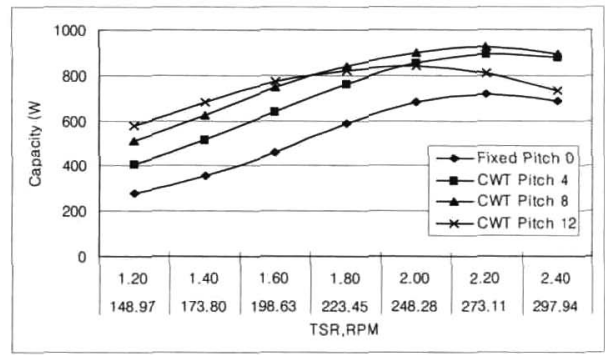

Fig. 8. Power generation according to rotating speed (Wind speed $13 \mathrm{~m} / \mathrm{s}$ )

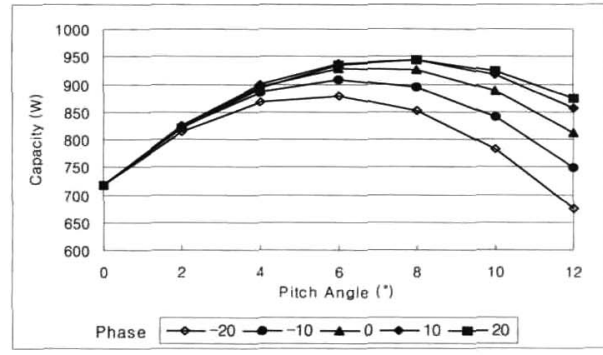

Fig. 10. Power generation according to pitch angle and phase angle (Wind speed $13 \mathrm{~m} / \mathrm{s}$ )

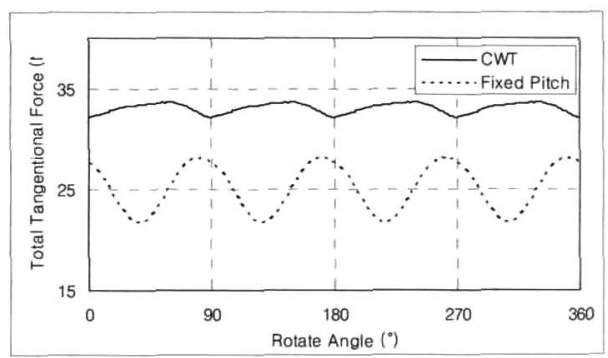

Fig. 12. Tangential force of whole rotor

Figure 7 shows power generation of CWT according to TSR (Tip Speed Ratio: the ratio of the blade-tip speed to the free-stream wind speed) for four pitch angles when wind speed is $4 \mathrm{~m} / \mathrm{s}$. Generated power increases as the rotor rotating speed faster until the peak position. The maximum power output value is produced at the pitch angle $8^{\circ}$ and TSR 2.2. Figure 8 shows the result at wind speed $13 \mathrm{~m} / \mathrm{s}$. The maximum power is generated at TSR 2.2 like the former case. When the rotor rotating speed is low, generated power is high at the large pitch angle. This phenomenon means that large torque can be obtained by large pitch angle at the starting condition.

Figure 9 shows the results of the generated power according to the change of pitch angle and phase angle under the condition of TSR 2.2 and wind speed $4 \mathrm{~m} / \mathrm{s}$. The maximum power is generated at the pitch angle $8^{\circ}$ and the phase angle $10^{\circ}$. Figure 10 shows the result at wind speed $13 \mathrm{~m} / \mathrm{s}$. The generated power is maximized at the pitch angle $8^{\circ}$ and the phase angle $10^{\circ}$ like the former case. The optimal pitch angle and phase angle according to the change of wind speed and wind direction should be applied in the design of the control mechanism of the cycloidal blade system. Figures 9 and 10 show that the phase angle should be maintained between $0^{\circ}$ and $10^{\circ}$ while the pitch angle maintains around $8^{\circ}$. However, these values can be changed if the rotor size of CWT and operating conditions are changed. Database in each case is also required. 


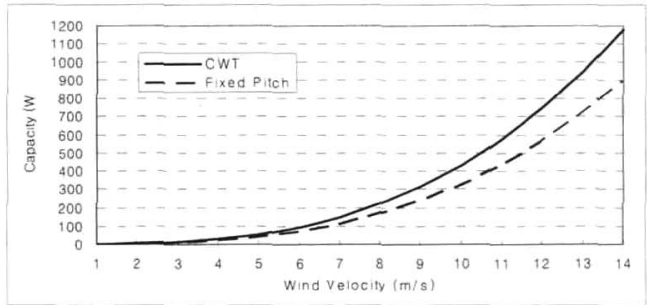

Fig. 13. Power generation according to wind speed (CWT and Fixed angle VAWT)

Figure 11 indicates the tangential force of each blade at wind speed $13 \mathrm{~m} / \mathrm{s}$, pitch angle $8^{\circ}$ and phase angle $10^{\circ}$ for CWT and fixed pitch wind turbine. Generally, upwind region - the position that azimuth angle is from $0^{\circ}$ to $180^{\circ}$ - generates large forces. In VAWT, wind speed at the downwind region is reduced to nearly half of that in the upwind region. Moreover, power generation is proportional to the cube of wind speed, therefore power output values of downwind region are much smaller than those of upwind region. The output power becomes even negative values at some positions. CWT makes larger force than fixed pitch wind turbine around the azimuth angle $90^{\circ}$.

Figure 12 shows the variation of the total tangential force during rotor rotation. In CWT, the difference between the maximum value and the minimum value of this force is about $5 \%$. This is relatively even power generation in wind turbine.

Overall force of CWT is larger than that of fixed pitch system. The performance is improved about 30\% at all wind speed region as shown in Fig. 13.

\section{Control Mechanism of CWT}

To realize the cycloidal motion, the mechanism controlling the pitch angle and the phase angle actively according to the change of wind direction and wind speed is required. Figure 14 shows a diagram of the control mechanism that consists of sensing part, control part and actuating part. Figure 15 displays the realized control devices of CWT. The wind indicator and the anemometer send the sensing information in analog voltage and pulse type. The microcontroller calculates appropriate pitch angle and phase angle from the sensed data to make PWM signal. Pitch control device is located at the top of the main shaft. The eccentricity point is moved by servo motor to change the maximum pitch angle. Phase control device is located at the bottom of the main shaft, connecting the pitch angle control part with the inner shaft. The shaft and the phase angle control servo motor are connected by the belt-pulley system, to change $\varepsilon$ in Fig. 4.

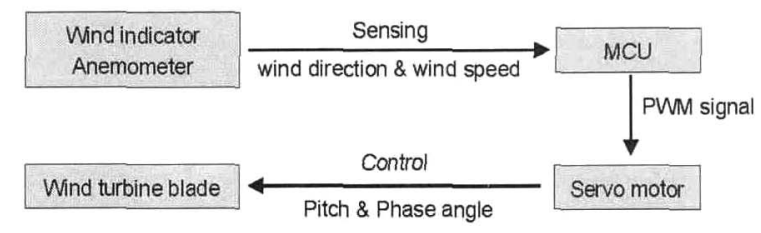

Fig. 14. Control mechanism diagram

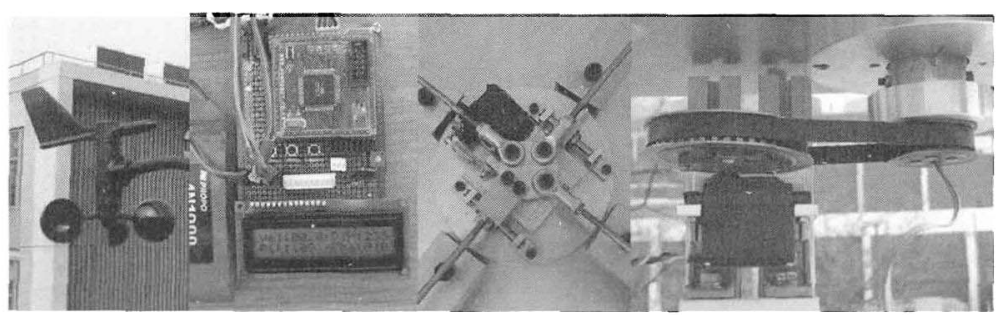

Fig. 15. Control devices of CWT 


\section{Concluding remarks}

In this paper, aerodynamic analysis and control mechanism design of the cycloidal wind turbine are described. Cycloidal blade system and active blade control system are adopted to the wind turbine for the performance improvement of a $\mathrm{H}$-Darrieus VAWT. The developing wind turbine equips four straight blades of $1 \mathrm{~m}$ span, and the rotor radius is $1 \mathrm{~m}$. The aerodynamic analysis shows that the cycloidal wind turbine is possible to generate approximately $30 \%$ higher power than the fixed pitch type VAWT by optimizing its pitch angle and phase angle according to wind direction and wind speed. The appropriate control mechanism to realize the cycloidal motion is designed with sensors, actuators and a microcontroller.

\section{References}

1. Paraschivoiu, I. "Wind Turbine Design with Emphasis on Darrieus Concept", Polytechnic International Press, 2002.

2. Hwang, I.S., Hwang, C.S., Min, S.Y., Jeong, I.O., Lee, Y.H. and Kim, S.J. "Efficiency Improvement of Cycloidal Wind Turbine by Active Control of Blade Motion", 16th International Conference on Adaptive Structures and Technologies, Paris, France, 9-12 October 2005.

3. Ham, N.D., Soohoo, P., Noll, R.B. and Drees, H.M. "Analytical and Experimental Evaluation of Cycloturbine Aerodynamic Performance," Terrestrial Energy Systems Conference, Orlando, Fla, 1979.

4. Wheatley, J.B. "Simplified Aerodynamic Analysis of the Cyclogiro Rotating-wing System", Technical Notes NACA No.467, 1933.

5. Kirsten, F.K. "Cycloidal propulsion in air", Bulletin No. 79, Engineering Experiment Station Series, University of Washington, 1935.

6. Boschma, J.H. "Modern Aviation Applications for Cycloidal Propulsion" „AIAA, Aircraft, Technology Integration and Operations Forum, Los Angeles, CA, 2001.

7. Yun, C.Y. "A New Vertical Take-off and Landing Aircraft with Cycloidal Blades System: Cyclocopter", Ph.D thesis, Seoul National University, Korea, 2004. 